\title{
Selecting Test Frequencies for Two-Tone Phase Plane Analysis of ADC's
}

\author{
Jerome Blair \\ Bechtel Nevada* \\ Las Vegas Nevada 89193-8521, USA \\ Phone: +1 702 295-2647, Fax: +1 702 295-2543 \\ Email: blairjj@nv.doe.gov,
}

\begin{abstract}
We show how to select the frequencies for a two-tone sinewave test of an analog to digital converter. The frequencies are selected in a manner to guarantee nearly uniform coverage of a selected ellipse in the phase plane. A proof that the selected frequencies provide the desired coverage is given..
\end{abstract}

Keywords - ADC testing, phase plane, two-tone tests, distortion compensation.

\section{INTRODUCTION}

A phase plane error model has long been recognized as a method of characterizing and correcting the dynamic errors of an analog to digital converter (ADC) and its sample-and-hold circuit ([1]-[8]). The phase plane error model assumes that the error at time, $t$, is a function of the value of the signal at $t$ and the value of the slew-rate (derivative with respect to time) of the signal at $t$. To apply the model we must supply a collection of input signals to the device under test (DUT) which have known values at all sample points and which give thorough coverage of the phase plane. Historically this has been accomplished with a number of sinewave signals. Each signal traces out an ellipse in the phase plane. By adjusting either the amplitudes or the frequencies (or both) of the sinewaves we get a sequence of signals that fill the part of the phase plane of interest. This is illustrated in figure 1.

\section{PRELIMINARIES}

Irons [9] recently discovered that the modeling can be accomplished with a single two-tone signal consisting of two sinewaves of the same amplitude and approximately equal frequencies. Figure $2 \mathrm{a}$ shows the quite good coverage of the phase plane that can be obtained with this approach. However, for some choices of the frequencies the coverage can be very poor, as is illustrated in figure $2 \mathrm{~b}$. In this paper we will show how to select frequencies that guarantee good phaseplane coverage.
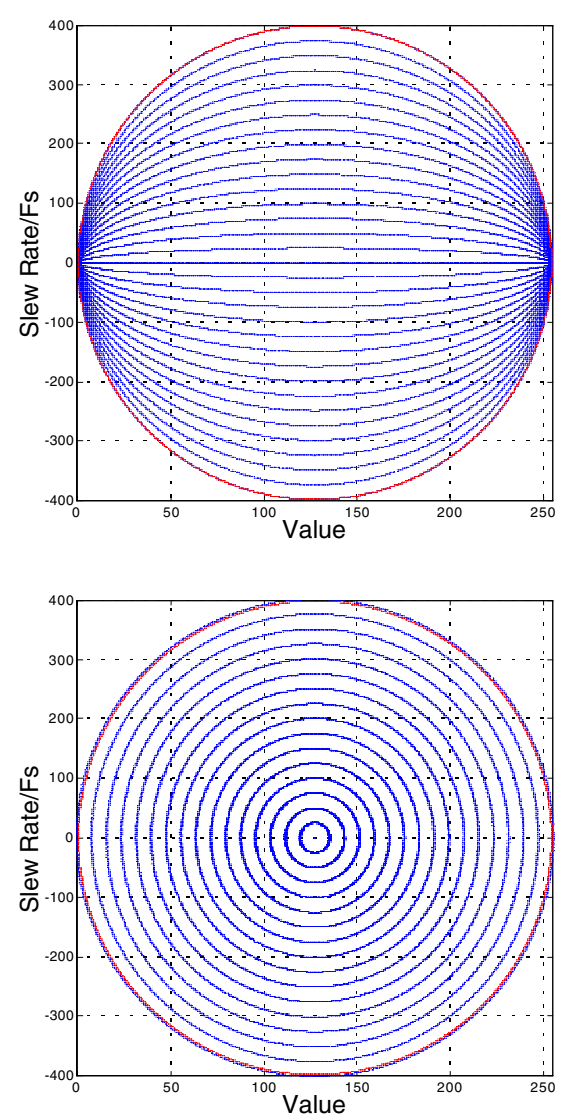

Figure 1. Both plots show phase-plane points for 16 signals, each sampled 4096 times. The outer circle is a full amplitude sinewave at the Nyquist frequency. The upper plot (a) is a full amplitude sinewave with varying frequency. The lower plot (b) is a Nyquist frequency sinewave with varying amplitude

\footnotetext{
* This work was supported by the U. S. Department of Energy, Nevada Operations Office, under contract No. DE-AC0896NV1178
} 


\section{RESULTS}

We assume a signal of the form

$s(t)=\frac{A}{2}\left(\sin \left(2 \pi f_{1} t+\phi_{1}\right)+\sin \left(2 \pi f_{2} t+\phi_{2}\right)\right)$

where $\phi_{1}$ and $\phi_{2}$ are arbitrary constants. Let $f_{\mathrm{s}}$ be the sampling frequency, $M=N^{2}$ be the number of signal values in a record, and $f_{0}=f_{\mathrm{s}} / M$. The two frequencies can then be selected by the rules:

$f_{1}=n_{1} f_{0}$,

$f_{2}=n_{2} f_{0}$,

$n_{2}=n_{1}-N$,

$\operatorname{GCD}\left(n_{1}, M\right)=\operatorname{GCD}\left(n_{2}, M\right)=1$.

where $n_{1}$ and $n_{2}$ are integers, and GCD is the greatest common divisor of its arguments. The requirement that the record length be a perfect square is necessary for the proof; however, we have found that using a non perfect square and using an integer, $N$, which is an approximate square root gives good phase-plane coverage. If $M$ is an even power of two $\left(M=2^{2 k}\right)$, then all the conditions of (2) are satisfied if $n_{1}$ is an odd integer.

The value for $A$ is chosen to cover the desired amplitude range. If the desired range is not symmetric about zero, a DC level will have to be added. Next, the value for $n_{1}$ is selected so that the maximum slew rate of the signal will be the desired maximum slew rate. Figure $2 \mathrm{a}$ is the result of applying this rule with $A=127$, a DC offset of 127, $M=4096$, and $n_{1}$ $=2047$.

The proof that this provides good coverage of the phase plane is divided into two parts. First the angle plane is introduced, and we show that the rules guarantee uniform coverage of a square in the angle plane. We then show that this uniform distribution in this square in the angle plane maps into an equally uniform distribution in the corresponding circle in the phase plane.

\section{ANGLE PLANE COVERAGE}

We will assume that $\phi_{1}$ and $\phi_{2}$ are zero. It should be clear from the proofs that their values are unimportant. The arguments of the two sine functions in (1) are given by the angles
$\theta_{\mathrm{i}}(k)=\operatorname{Mod}\left(2 \pi n_{i} k / M, 2 \pi\right)=$

$\frac{2 \pi}{M} \operatorname{Mod}\left(n_{i} k, M\right)$, for $i=1,2$.
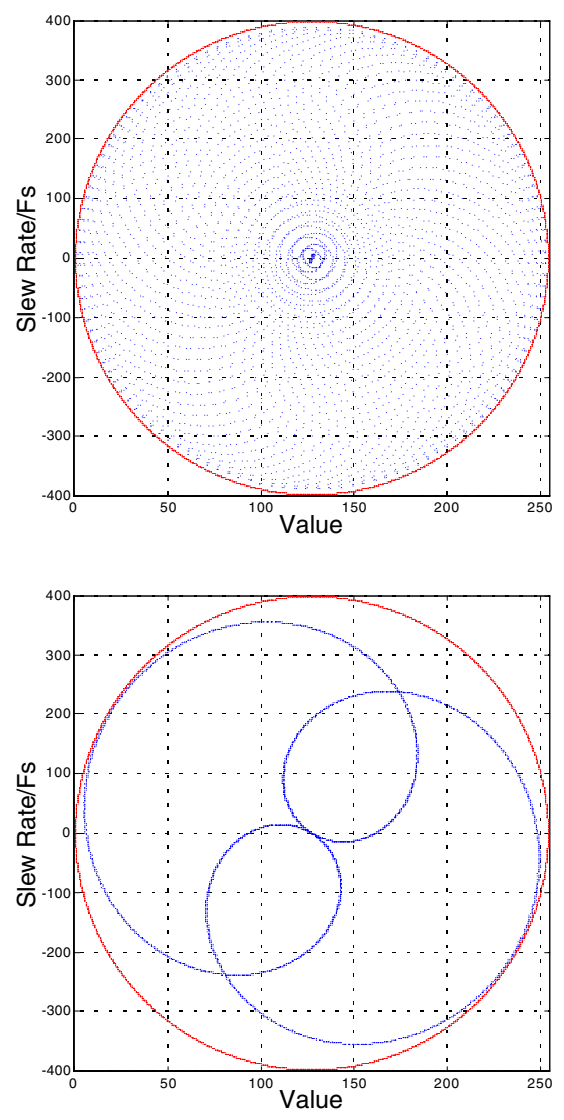

Figure 2. Both plots are the phase plane points of a two-tone signal sampled 4096 times. The outer circle is a full amplitude sinewave at Nyquist frequency. In the upper plot (a) the sinewaves were in the frequency bins 2047 and 1983 (=2047 - 64.) In the lower plot (b) the frequency bins were 2047 and 2045 .

It is more convenient to represent the angles with the variables

$T_{i}(k)=\operatorname{Mod}\left(n_{i} k, M\right)$, for $i=1,2$.

$\operatorname{Mod}(x, y)$ is the remainder when $x$ is divided by $y$. Because of the GCD conditions in (2), as $k$ varies from 0 to $M-1, T_{i}(k)$ will take on every integer value between 0 and $M-1$ exactly once. We want to show that the points $\left(T_{1}(k), T_{2}(k)\right)$ uniformly cover the square $[0, M-1] \times[0, M-1]$ in the angle plane. Let 
$b_{i}(k)=T_{i}(k) / N$ (integer divide.)

Each $b_{i}(k)$ takes integer values between 0 and $N-1$. The set of values of $\left(T_{1}(k), T_{2}(k)\right)$ for which $\left(b_{1}(k), b_{2}(k)\right)$ has a constant value is an $\mathrm{N}$ by $\mathrm{N}$ sub square of the square $[0, M-1] \times[0, M-1]$ in the angle plane. As $\left(b_{1}(k), b_{2}(k)\right)$ takes on its $\mathrm{N}^{2}$ possible values these disjoint sub squares cover the entire larger square. We will show that as $k$ varies from 0 to $M-1$ the point $\left(T_{1}(k), T_{2}(k)\right)$ appears in each of these smaller squares exactly once.

It will be found useful to introduce two additional functions, $\Delta T$ and $\Delta b$, given by

$$
\begin{aligned}
& \Delta T(k)=\operatorname{Mod}\left(T_{1}(k)-T_{2}(k), M\right), \text { and } \\
& \Delta b(k)=\operatorname{Mod}\left(b_{1}(k)-b_{2}(k), N\right) .
\end{aligned}
$$

From (4) and (6) it follows that

$$
\Delta T(k)=\operatorname{Mod}(N \times k, M) .
$$

From (8) and (5) we obtain

$$
\Delta b(k)=\operatorname{Mod}(k, N)
$$

where we have used the fact that because $N$ is a divisor of $M$, $\operatorname{Mod}(\operatorname{Mod}(k, M), N)=\operatorname{Mod}(k, N)$.

We now assume two integers $k_{\mathrm{a}} \leq k_{\mathrm{b}}$, both between 0 and $M$ 1 for which

$$
b_{1}\left(k_{a}\right)=b_{1}\left(k_{b}\right) \text {, and } b_{2}\left(k_{a}\right)=b_{2}\left(k_{b}\right) \text {, }
$$

and show that $k_{\mathrm{a}}=k_{\mathrm{b}}$. This implies that each of the small squares is occupied exactly once. From (10) is clear that $\Delta b\left(k_{\mathrm{a}}\right)=\Delta b\left(k_{\mathrm{b}}\right)$, which implies that $\operatorname{Mod}\left(k_{\mathrm{b}}-k_{\mathrm{a}}, N\right)=0$, which means that $k_{\mathrm{b}}=k_{\mathrm{a}}+p N$, with $p$ and integer satisfying $0 \leq p \leq N-1$. Substituting this into (10) using (5) and (4) gives

$$
\operatorname{Mod}\left(n_{1} k_{a}, M\right) / N=\operatorname{Mod}\left(n_{1} k_{a}+n_{1} p N, M\right) / N .
$$

Since two quantities yield the same quotient when divided by $N$, the absolute value of their difference must be less than $N$, or

$$
\left|\operatorname{Mod}\left(n_{1} p N, M\right)\right|<N
$$

$\operatorname{Mod}\left(n_{1} p N, M\right)=n_{1} p N-q M=n_{1} p N-q N^{2}$,

for some integer, $q$. This is divisible by $N$. Being divisible by $N$ and having absolute value less than $N$ means the number is zero, or

$n_{1} p=q N$.

This implies that $n_{1} p$ is divisible by $N$. Since, by the GCD condition in (2), $n_{1}$ has no common factors with $M$, and all factors of $N$ are also factors of $M, n_{1}$ has no common factors with $N$. This means that $p$ is divisible by $N$. Since $p$ is between 0 and $N-1$, it must be zero. Thus, from the comments following (10), $k_{\mathrm{a}}=k_{\mathrm{b}}$.

\section{PHASE PLANE COVERAGE}

We now show that the nearly uniform angle plane coverage leads to nearly uniform phase plane coverage. We have for the signal and its derivative

$$
s(t)=\frac{A}{2}\left(\sin \left(2 \pi f_{1} t\right)+\sin \left(2 \pi f_{2} t\right)\right)=\frac{A}{2}\left(\sin \left(\theta_{1}\right)+\sin \left(\theta_{2}\right)\right)
$$

, and

$$
\begin{aligned}
& s^{\prime}(t)=\pi A\left(f_{1} \cos \left(2 \pi f_{1} t\right)+f_{2} \cos \left(2 \pi f_{2} t\right)\right) \\
& \cong \pi A f\left(\cos \left(\theta_{1}\right)+\cos \left(\theta_{2}\right)\right)
\end{aligned}
$$

where $f=\left(f_{1}+f_{2}\right) / 2$. The last approximation in (16) is valid, because the difference between the two frequencies is typically small compared to the frequencies. If $f_{1}$ is approximately the Nyquist frequency, then $f_{1}-f \cong f / N$.

We will use as the phase plane variables $x=s(t)$, and $y=$ $s^{\prime}(t) / 2 \pi f$, so that both variables will range from $-A / 2$ to $+A / 2$. The bold circle in figure 3 shows the locus of points in the phase plane with $\theta_{1}$ held constant and $\theta_{2}$ varying from 0 to 2 $\pi$. This is a circle with radius $A / 2$ centered on the circle of radius $\mathrm{A} / 2$ that is centered at the origin (shown with dashed line.) The center depends on the angle $\theta_{1}$ as shown in the diagram. The results obtained show that for a fixed value of $\theta_{1}$ (i.e. a fixed value of $b_{1}$ in (5)) we will obtain $N$ points in the phase plane approximately uniformly distributed around the outer circle of radius $A / 2$. This pattern is repeated $N$ times with the centers of the circles being approximately uniformly distributed around the inner circle. This establishes that the criteria in (2) provides good coverage of the phase plane, at least for the case in which the phase angles, $\phi_{1}$ and $\phi_{2}$, in (1) are zero.

By definition 
Non-zero values for $\phi_{1}$ and $\phi_{2}$ merely add constants to the angles appearing in (15) and (16). This just shifts the angles of the $N$ points on each outer circle, in the case of $\phi_{2}$, and shifts the angles on the inner circle of the centers of these circles in, the case of $\phi_{1}$.

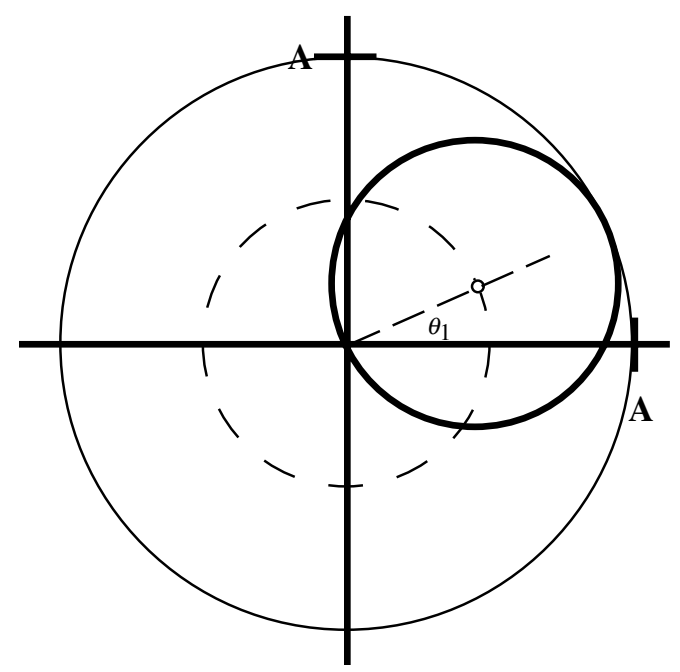

Figure 3. The bold circle shows the path traversed in the phase plane with $\theta_{1}$ constant and $\theta_{2}$ varying from 0 to $2 \pi$.

\section{CONCLUDING REMARKS}

We have established that one can get good coverage of a specified ellipse in the phase plane with a single two-tone signal obtained by adding two sinewaves of equal amplitude and approximately equal frequency. The criteria given for selecting record lengths and frequencies are more stringent than are required but are easy to meet.
There are some shortcomings worth noting in what we have presented here. Our method of proof seems to not yield to application to record lengths that are not perfect squares. The proof is also not intuitive. The most serious shortcoming is that we have not yet analyzed how accurately the specified frequencies must be achieved. It is hoped that other authors will take up these matters

\section{ACKNOWLEDGEMENTS}

The author would like to thank Fred Irons for pointing out this interesting problem and for supplying the software with which to investigate the problem and which was used to generate figure 2 .

\section{References}

[1] J. J. Blair, "The characterization and measurement of nonlinearities in transient measuring systems," IEEE Trans. Instrum. Meas., vol. 21, pp 500-504, 1972.

[2] F. H. Irons and T. A. Rebold, "Characterization of high-frequency analog-to-digital -converters for spectral analysis applications", MIT Lincoln Laboratory Project Report AST-2, November 1986.

[3] J. P. Deyst, J. J. Vytal, P. R. Blasche and W. M. Siebert., "Wideband distortion compensation for bipolar flash analog-to-digital converters" IEEE-IMTC92 Conference Record, pp 290-294.

[4] D. M. Hummels, F. H. Irons, R. Cook, and Y. Papantonopoulos, "Characterization of ADCs using a non-iterative procedure", IEEE Intl Symp on Ckts \& Sys, May 1994, London, England pp 5- 8, Vol.2.

[5] F. H. Irons, D. M. Hummels, and C. A. Zoldi, "ADC architectural diagnostic testing procedures", Proc Gov't MicroCircuit Appl Conf (GOMAC96), pp 79-80, Kissimmee, FL, Mar 1996.

[6] D. M. Hummels, I. N. Papantonopoulos, and F. H. Irons "Identification of error mechanisms in a folding and interpolating ADC", Proc IEEE Int'1 Symp on Ckts \& Sys, Atlanta, GA, pp 176-179, Vol.4, May 1996.

[7] Kelso, GM, Hummels,DM \& Irons, FH, "Fast Compensation of Analog-to-Digital Converters", Proc IEEE Instr \& Meas Tech Conf (IMTC99), Venice, Italy, pp 1295-1298, May 1999. IEEE Cat.No: 99CH36309- ISBN: 0-7803-5276-9

[8] J. H. Larrabee, F. H. Irons, and D. M. Hummels, "Using sinewave histograms to estimate analog-to-digital converter dynamic error functions", IEEE Trans. Instrum. Meas., Vol.47, pp 1448-1456, Dec1998.

[9] F. H. Irons, private communication, 1998 